\title{
Design and Experimental Implementation of Easily Detachable Permanent Magnet Reluctance Wheel for Wall-Climbing Mobile Robot
}

\author{
Jin-Ho Kim*, Se-Myung Park, Je-Hoon Kim, and Jae-Yong Lee \\ School of Mechanical Engineering, Yeungnam University, Gyeongbuk 712-749, Korea
}

(Received 31 May 2010, Received in final form 27 July 2010, Accepted 3 August 2010)

\begin{abstract}
In this paper, we propose a new design of the permanent magnet reluctance wheel which will make it possible to attach the robot to a vertical plane and move it. In the newly suggested design, a permanent magnet is utilized to enhance the adhesive force during attachment, and an electromagnet is produced to weaken the magnetic field of the permanent magnet and reduce the adhesive force for easier detachment of wheels from steel plates. To characterize the performance of this new wheel design, a 3-D finite element analysis is executed using a commercial FE program. The results show that the adhesive force is reduced effectively by the electromagnet which flows in the reverse direction of the magnetic loop of the permanent magnet when the current is supplied to the coil.
\end{abstract}

Keywords : detachment, inducing pin, mobile robot, permanent magnet reluctance wheel

\section{Introduction}

In order to build a large structure such a ship, we need safety equipments to allow workers to work on an inclined or a vertical plane, but it needs many hours and costs, and it has many casualties. Thus, researchers have been developing devices that will enable mobile robots to operate on inclined or vertical walls.

The wall-climbing robots have been widely used to attach on the wall such as the suction types. Luk et al. developed a 4-legged articular "Nuro robot", which legs and body had suction cups, and Bahr et al. measured the attaching force of the suction cup on horizontal and vertical walls [1-2]. Wang Yan introduced new suction plates with improved efficiency and tolerance loads [3]. Also, Nishi reported various types of suction cups [4]. These types can be utilized regardless of the wall material. Furthermore, they have good attaching force-weight ratio and the attaching force can be easily controlled. However, fast motion of the robots cannot be obtained when suction cups adhere to the wall. In addition, supplementary devices are required to adhere and control the suction cups. On the other hand, Hirose introduced a robot named "Disk Rover" [5]. It has inclined magnetic disks which

*Corresponding author: Tel: +82-53-810-2441

Fax: +82-53-810-4627, e-mail: jinho@ynu.ac.kr can attach and rotate on the wall and produce the driving force to move forward.

Among the devices developed for mobile robots, the most advanced one is the permanent magnet wheel introduced by S.C. Han et al. [6]. In the suggested permanent magnet wheel assembly, the residual magnetic field of the permanent magnet creates the adhesive force during attachment while the inducing metal pins change the magnetic flow and weaken the adhesive force during detachment.

Fig. 1 shows the structure and operation principle of the inducing pin type permanent magnet wheel assembly. A magnetic closed loop is constructed between the permanent magnet and the steel plate, and this loop creates the attaching force of the wheel (Fig. 1(a)). If we insert the inducing pins into the holes of the wheel, some of the

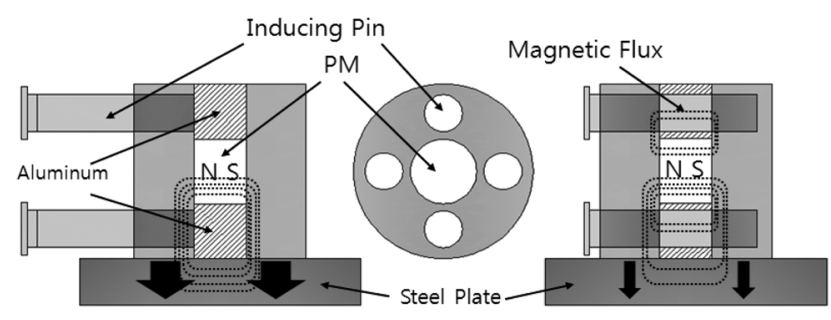

(a)

(b)

Fig. 1. Structure and operation principle of inducing pin type permanent magnet wheel (a) attaching mechanism (b) detaching mechanism using inducing pins [7]. 
Table 1. Required force to separate the inducing pins from the holes of pin type magnet wheels according to the number of inducing pins.

\begin{tabular}{ccccc}
\hline \hline Number of pins & 1 & 2 & 3 & 4 \\
\hline Force & $46 \mathrm{~N}$ & $37 \mathrm{~N}$ & $30 \mathrm{~N}$ & $24 \mathrm{~N}$ \\
\hline
\end{tabular}

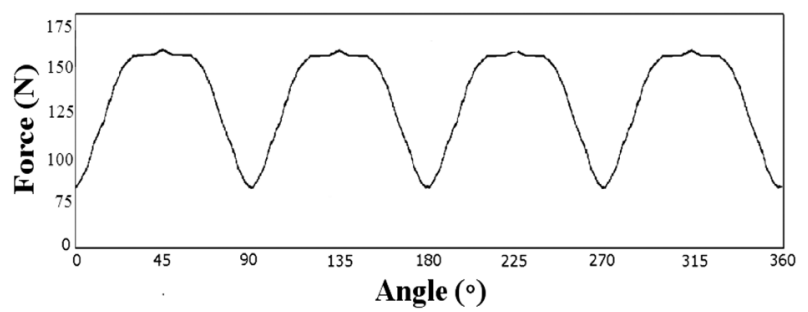

Fig. 2. Magnetic attaching force according to rotation of the wheel.

magnetic flux of the permanent magnet is induced to flow through the pins, weakening the attaching force (Fig. 1(b)). Accordingly, the permanent magnet wheel can have smaller weight and greater mobility than suction cups.

The permanent magnet wheel, however, has critical drawbacks. First, the inducing pins are difficult to separate from holes of the magnet wheels after work completion, although inducing pins can be easily inserted into the holes. Table 1 shows the required force to separate the pins from the holes of the pin type magnet wheel after work completion according to the number of inducing pins.

Second, the magnetic attaching force fluctuates because of the change of the positions of the holes as the wheels rotate. Fig. 2 shows magnetic attaching force according to rotation of wheel.

Accordingly, a constant wheel speed cannot be achieved. Therefore, Kim et al. suggests the concept of the permanent magnet reluctance wheel to overcome the drawbacks of the inducing-pin type magnet wheel

In this paper, we demonstrate the performance of the novel permanent magnet reluctance wheel by experiment and compare the results with FE simulation. In the suggested design, a permanent magnet is utilized to enhance the adhesive force during attachment of the wheels on the wall, while an electromagnet is introduced to weaken the magnetic field of the permanent magnet and thereby reduce the adhesive force for easier detachment of wheels from steel plates.

\section{Permanent Magnet Reluctance Wheel}

\subsection{Structure and operation principle}

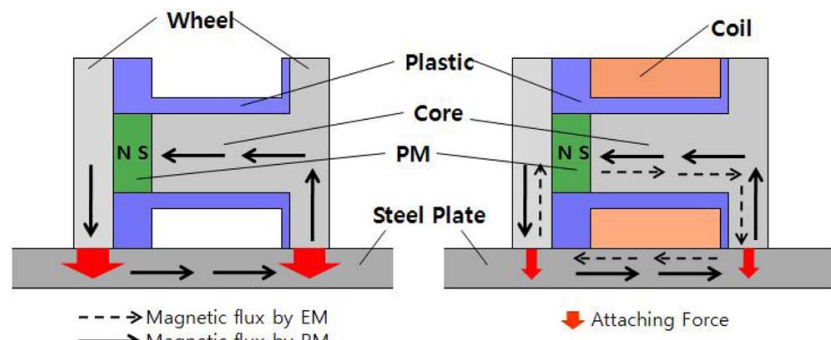

(a)

(b)

Fig. 3. Structure and operation principle of permanent magnet reluctance wheel (a) attaching mechanism (b) detaching mechanism using inducing magnetic flux of electromagnets.

Fig. 3 shows the structure and operation principle of the permanent magnet reluctance wheel. It consists of a pair of disk wheels, permanent magnet, electromagnetic coil and steel core. In Fig. 3, the solid arrows show the magnetic flux generated by the permanent magnet, and the dotted arrows show the flux generated by the electromagnet. At attachment, the magnetic flux of the permanent magnet forms a closed magnetic loop to the steel plate which results in the attachment force. To reduce the attachment force, the electromagnetic coil is energized and the magnetic flux of the electromagnet flows in the reverse direction of the magnetic loop of the permanent magnet. Accordingly, the magnetic flux partially cancels the total magnetic force, resulting in the decrease of the magnetic force.

Compared with the inducing pin type magnet wheel, the permanent magnet reluctance wheel has two main advantages. First, it provides constant magnetic attaching force because it has no holes on the wheel. Second, the attaching force of the permanent magnet reluctance wheel is easy to control by amount of current supplied to the electromagnetic coil.

\subsection{Finite element analysis}

To characterize the enhanced performance of the newly proposed design, finite element analysis is performed using a commercial electromagnetic analysis program, Maxwell. We perform 3-D static finite element analysis using tetrahedron elements of which the number of elements is 48729. For the comparison of the magnet wheel with 4 inducing pins with the permanent magnet reluctance wheel, respective models are created. The disk wheel and permanent magnet in these models are of the same size, but the inducing pin type magnet wheel has pin holes in the wheel disk. Fig. 4(a) and Fig. 4(b) show the specifications of the models, respectively. Magnetic B-H curve is assigned to the wheel disk and core and the magnetic properties 


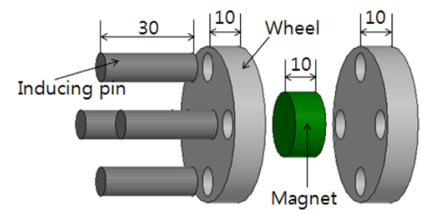

(a)

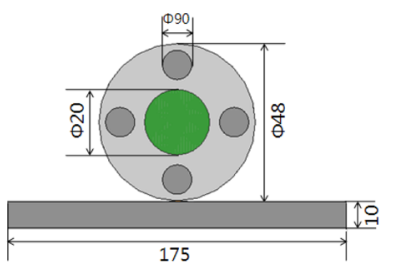

(a)
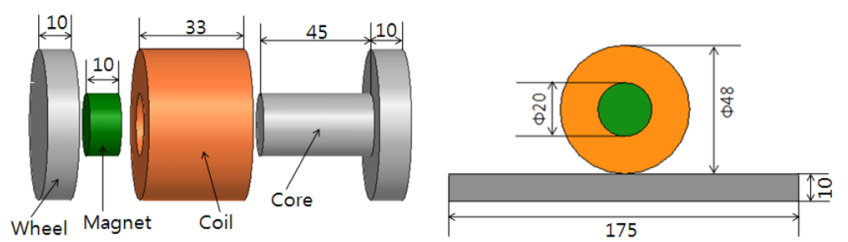

(b)

Fig. 4. FE model specification.

Table 2. Magnetic Properties of N25UH5.

\begin{tabular}{cc}
\hline \hline Relative Permeability $(\mathrm{Mu})$ & 1.099 \\
Residual Induction $(\mathrm{Br})$ & $0.996 \mathrm{~T}$ \\
Coercivity $(\mathrm{Hc})$ & $-732000 \mathrm{~A} / \mathrm{m}$ \\
\hline
\end{tabular}

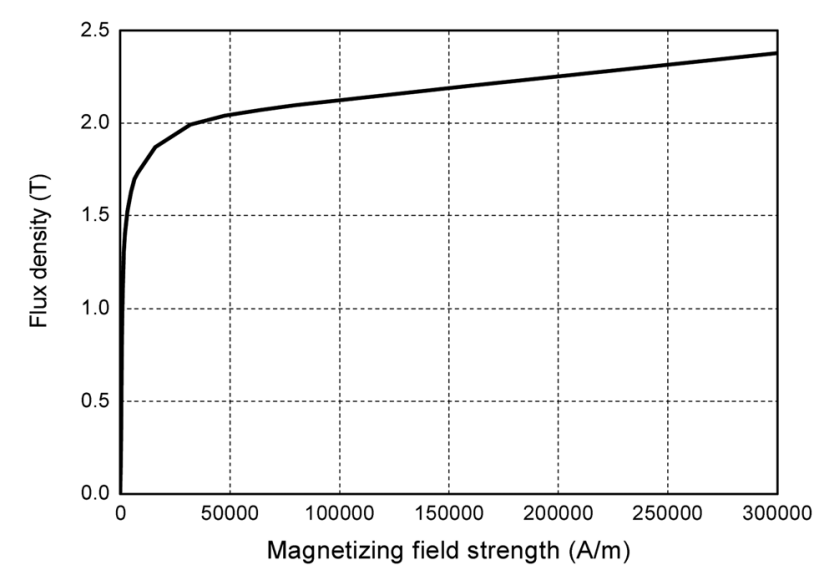

Fig. 5. Initial magnetization curves of 1010 steel.

of the N25UH5 magnet are assigned to the permanent magnet. Table 2 shows the magnetic properties of N25UH5 and Fig. 5 shows the nonlinear magnetization curve of 1010 steel.

Fig. 6 shows the magnetic attaching force of the permanent magnet reluctance wheel according to the current applied to the electromagnet. A magnetic force of $174 \mathrm{~N}$ is produced but reduces by $4.88 \mathrm{~N}$, on average, per $90 \mathrm{~A} \cdot \mathrm{t}$ of current applied to the electromagnet.

Fig. 7 shows the plot of magnetic flux in permanent magnet reluctance wheel according to current applied to electromagnet. We verify that the electromagnet reduces the magnetic flux flowing from the magnet to the steel

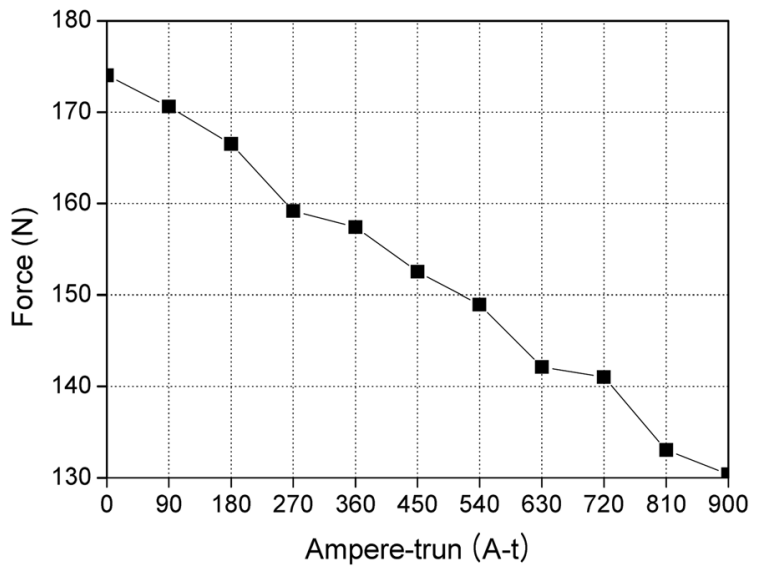

Fig. 6. Magnetic attaching force according to current applied to electromagnet of permanent magnet reluctance wheel in finite element analysis.

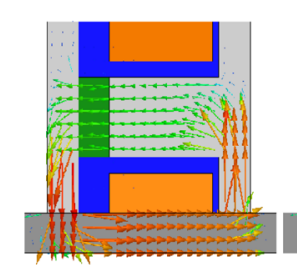

(a) $0 \mathrm{~A} \cdot \mathrm{t}$

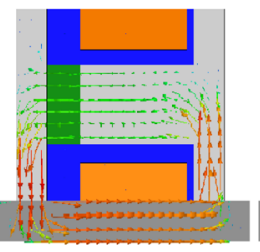

(b) $450 \mathrm{~A} \cdot \mathrm{t}$

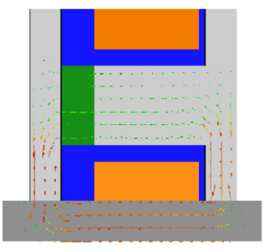

(c) $900 \mathrm{~A} \cdot \mathrm{t}$
Fig. 7. Plot of magnetic flux according to current applied to electromagnet of permanent magnet reluctance wheel.

plate.

The force of the permanent magnet reluctance wheel is constant, regardless of the wheel rotation, while the pin type wheel oscillates due to the position change of the holes.

\section{Experiments of Permanent Magnet Reluctance Wheel}

To demonstrate the detaching performance of the electromagnetic coil in the permanent magnet reluctance wheel, a permanent magnet reluctance wheel with 90 coil-turns is constructed for experiment, as shown in Fig. 8. We energize the electromagnetic coil, increasing the current from 1 to 10 by $1 \mathrm{~A}$.

In Fig. 8, the current supplier energizes the permanent magnet reluctance wheel, which then detaches easily, and the load cell is used to measure the attaching force, which is applied when the detaching force test bar turns upside to detach the permanent magnet reluctance wheel. Fig. 9 shows the components of the permanent magnet reluctance wheel. The coil AWG 15 of diameter $1.45 \mathrm{~mm}$ is wound around of the plastic covering the core. 


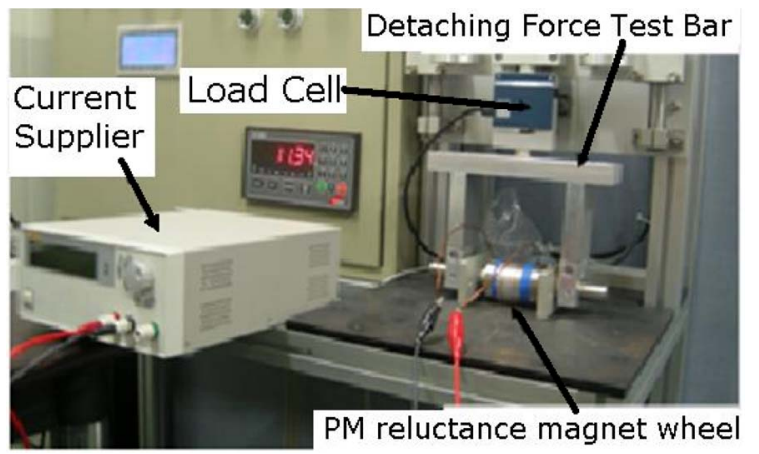

Fig. 8. Performance test equipment of permanent magnet reluctance wheel.

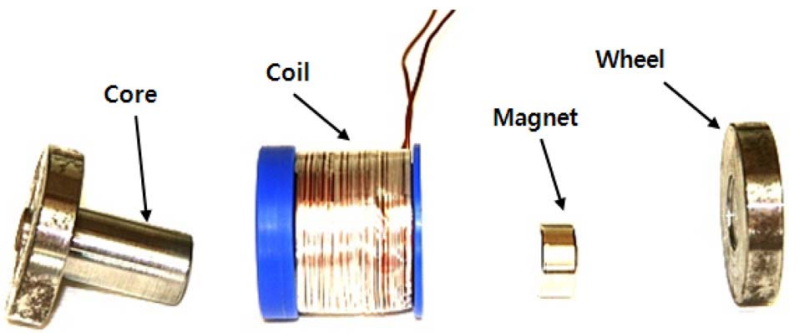

Fig. 9. Components of the permanent magnet reluctance wheel.

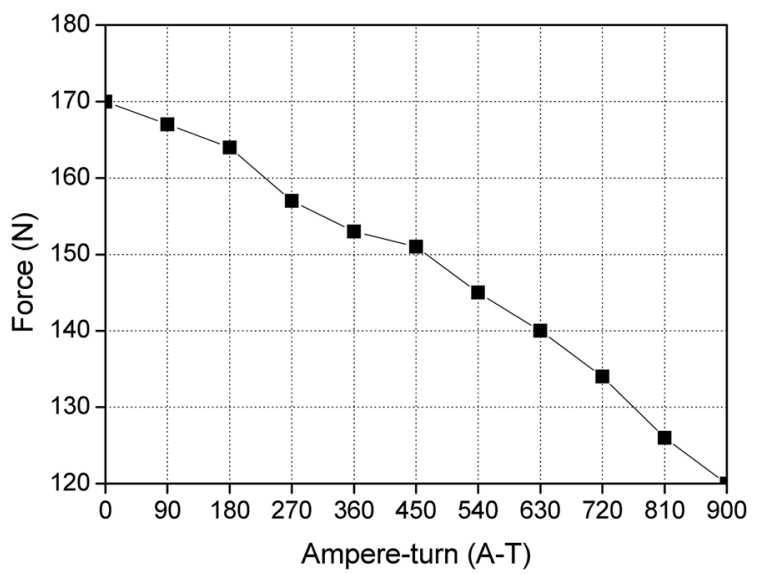

Fig. 10. Magnetic attaching force according to current increase to permanent magnet reluctance wheel in experiment.

Experimental results are shown in Fig. 10. As the current supplied to the coil is increased, the magnetic force is reduced. For the case of 90 coil-turns, the magnetic force is reduced as the induced current is increased.

To test the demagnetization of the permanent magnet due to current, the magnetic attaching force is measured with the load cell when the supplied current is removed.
A magnet attaching force of $170 \mathrm{~N}$ is measured.

\section{Conclusions}

A novel design of the permanent magnet reluctance wheel is introduced and built in this study. To overcome the inherent drawbacks of the pin type wheel, both the permanent magnet and the electromagnet were simultaneously used in the newly designed magnet-wheel system. The attaching force of the permanent magnet reluctance wheel was easy to reduce, unlike that of the pin type wheel, which inducing pins were difficult to pull out from the holes.

A model of the permanent magnet reluctance wheel was designed simply for 3-dimensional finite element analysis, which was carried out by the Maxwell electromagnetic analysis software. The result of the analysis demonstrated that the permanent magnet reluctance wheel produced a constant magnetic attaching force, regardless of the wheel rotation. The energized electromagnet reduced the attaching force effectively. This new design of permanent magnet reluctance wheel can be employed to mobile robot for working on inclined or vertical plane such a huge ship, which saves high labor costs due to the safety problem.

\section{Acknowledgement}

This research was supported by Yeungnam University research grant in 2010.

\section{References}

[1] B. L. Luk, A. A. Collie, and J. Billingsley, Proceedings of the 1991 IEEE International Conference on Robotics and Automation, Sacramento, California, 2342 (1991).

[2] B. Bahr, Y. Li, and M. Najafi, Computer Elect. Eng. 22, 193 (1996).

[3] Y. Wang and S. Liu, Proceedings of the 1999 IEEE International Conference on Robotics and Automation, Detroit, Michigan, 1207 (1999).

[4] A. Nishi, Computers Elect. Eng. 22, 123 (1996).

[5] S. Hirose, and H. Tsutsumitake, Proceedings of the 1992 IEEE/RSJ International Conference on Intelligent Robots and System, 2074 (1992).

[6] S. C. Han, J. H. Kim, and H. C. Yi, International Journal of Precision Engineering and Manufacturing 10, 143 (2009). 